\section{Life-Threatening Severe Hyperkalemia Presenting Electrocardiographic Changes}

\section{Abstract}

Hyperkalemia is a common and potential life-threatening electrolyte disorder in patients presenting to the emergency setting. It is known that renal insufficiency may abate the toxic effects of hyperkalemia on electrocardiographic abnormality formation. Herein, I have reported a case of severe hyperkalemia associated with renal insufficiency in a woman patient that presented to the emergency department with weakness and hypotension. Initial electrocardiogram showed accelerated AV junctional rhythm. Incipient treatment for hyperkalemia was begun and afterwards hemodialysis was performed for patient. Then, electrocardiogram was repeated that primary changes were removed and vital sign was stable. Eventually, it is better that hemodialysis starts from commencement for patients with life-threatening severe hyperkalemia associated with acute or chronic renal failure.

Keywords: Severe hyperkalemia; Renal insufficiency; Hemodialysis
Seyed Farshad Heidari

Mazandaran University of Medical Sciences, Sari, Mazandaran, Iran

Corresponding author:

Seyed Farshad Heidari

” s.f.heidari@gmail.com

Mazandaran University of Medical Sciences, Sari, Mazandaran, Iran.

Tel: 988338224670

Fax: 9188317379

Citation: Heidari SF. Life-Threatening Severe Hyperkalemia Presenting Electrocardiographic Changes. J Intensive \& Crit Care 2016, 2:3.

Received: August 09, 2016; Accepted: August 16, 2016; Published: August 23, 2016

\section{Introduction}

Hyperkalemia is a common and potential life-threatening electrolyte disorder in patients presenting to the hospital setting. The prevalence of hyperkalemia varies in diverse studies, with estimates ranging between $1 \%$ to $10 \%$ of hospitalized patients [1]. Mild hyperkalemia (serum potassium concentrations of 5.5-6.5 mmol/L) has been associated with tall peaked $\mathrm{T}$ waves; moderate hyperkalemia, with loss of $P$ waves (6.5-7.5 $\mathrm{mmol} / \mathrm{L}$ ) and QRS complex widening (7.5-8.0 mmol/L); severe hyperkalemia (8.0-10.0 mmol/L), with ventricular arrhythmias and asystole [2]. Other electrocardiographic manifestation has been reported in the literature [3]. It can cause lethal arrhythmias, flaccid paralysis, and respiratory difficulty. When changes detected on electrocardiogram, severe hyperkalemia should be treated urgently, even before laboratory confirmation, especially in critically ill patients $[4,5]$. It is known that chronic renal failure may detract the toxic effects of hyperkalemia on electrocardiographic abnormality formation $[6,7]$.

In this study has been reported a case of life-threatening severe hyperkalemia with electrocardiogram changes that rapidly resolved this changes with treatment.

\section{Case Report}

A 76 year old woman patient presented to the emergency room with progressive generalized weakness since 12 days ago. Patient was anuric from 2 days ago. Associated symptoms includes anorexia, nausea, vomiting and confusion. She had a history of chronic renal insufficiency and hypertension for 3 years ago and also chronic heart failure from 3 months ago. There was no history of dialysis during this period. Also, drug history was negative. Patient was ill in appearance. In the emergency room, initial blood pressure was $80 / 60 \mathrm{mmhg}$ and pulse rate was 70 per min. Other examinations were normal. The electrocardiogram showed accelerated AV junctional rhythm (Figure 1). Metabolic acidosis ( $\mathrm{pH}$ of 7.12 and base excess of $-11.6 \mathrm{mmol} / \mathrm{L}$ ) was noted through arterial blood gas analysis. Routine laboratory studies reveal severe hyperkalemia (serum potassium, $8.4 \mathrm{mmol} / \mathrm{L}$ ) and renal dysfunction (urea, $342 \mathrm{mg} / \mathrm{dl}$ and serum creatinine, 9.5 $\mathrm{mg} / \mathrm{dL}$ ). Serum levels of cardiac enzymes were within normal limit. She was promptly treated with intravenous fluid, $20 \mathrm{~mL}$ of intravenous $10 \%$ calcium gluconate, 10 units of intravenous insulin with $50 \%$ dextrose and $84 \mathrm{meq} / \mathrm{l}$ sodium bicarbonate intravenously. Then, temporary hemodialysis was performed for $3 \mathrm{~h}$ through a right internal jugular vein instantly before preparing results of laboratory tests. After $2 \mathrm{~h}$ of management, a repeat electrocardiography was normal without primary changes (Figure 2), his vital sign was stable with blood pressure 110/65 $\mathrm{mmHg}$, and serum potassium level was $4.3 \mathrm{mmol} / \mathrm{L}$. Metabolic acidosis was partially improved with $\mathrm{pH}$ of 7.29 and base excess of $-3.5 \mathrm{mmol} / \mathrm{L}$. Her renal function was progressively improved with serum creatinine, $2.3 \mathrm{mg} / \mathrm{dL}$. 


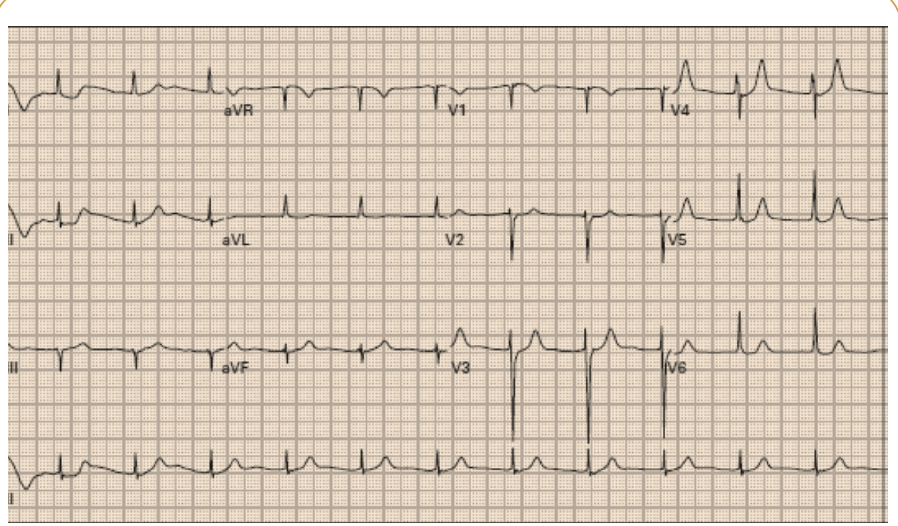

Figure 1 The electrocardiographic changes on admission of patient that showed the absence of $P$ waves and junctional rhythm.

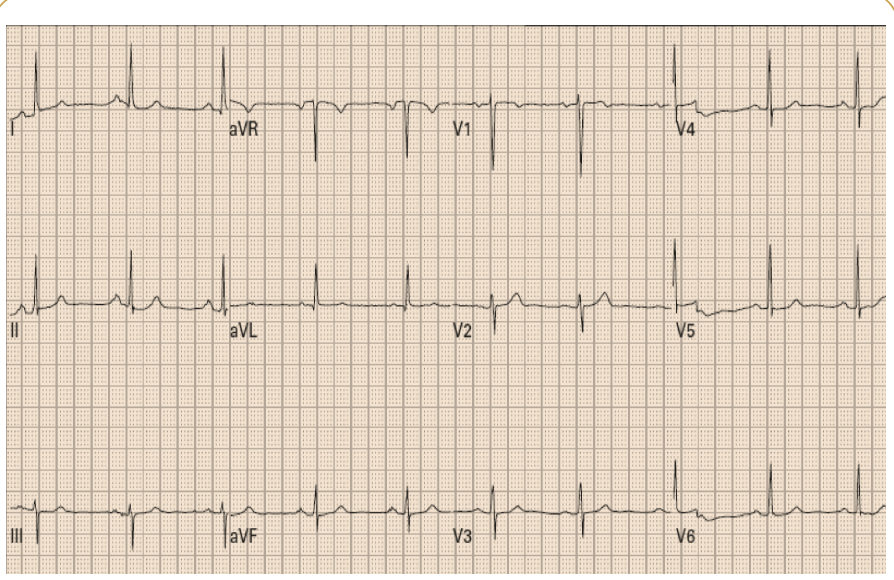

Figure 2 A repeat electrocardiograph was performed after $2 \mathrm{~h}$ of management for hyperkalemia that was normal and primary changes was removed.

\section{Discussion}

Hyperkalemia is a life-threatening electrolyte abnormality resulting in a perilous cardiacarrhythmia. The electrocardiographic manifestations of hyperkalemia include the peaked $\mathrm{T}$ waves generally considered the earliest sign, progressive prolongation of the PR and QRS intervals and decreased amplitude and eventual loss of the $P$ waves. As serum potassium level rises, sinoatrial and atrioventricular conduction was blocked, causing escape rhythms. This is followed by widening of the QRS complex and merging with the T wave to form a "sine-wave" appearance, which may result in ventricular fibrillation or asystole [8]. In this case, typical electrocardiographic features of severe hyperkalemia ( $>8.0 \mathrm{mmol} / \mathrm{L}$ ) including the absence of $\mathrm{P}$ waves and junctional escape rhythm was noted.

Prompt treatment of hyperkalemia was imperative to prevent further development of fatal cardiac arrhythmia. First, intravenous calcium gluconate should be infused in patients with electrocardiographic changes to stabilize membrane potential [9]. Then insulin with dextrose infusion, intravenous or nebulized beta-adrenergic agonists (salbutamol), or intravenous sodium bicarbonate can be used to increase potassium shift from extra- to intracellular space [10]. In this case, immediately, performed empirical treatments of hyperkalemia based on his electrocardiographic manifestations before achieving results of serum electrolyte concentration. Prompt inception with intravenous calcium, insulin with glucose, sodium bicarbonate and short-term hemodialysis completely improved hyperkalemia and recovered junctional rhythm with hemodynamic instability within the first few hours.

It is also important to identify and eliminate underlying causes or precipitating factors of hyperkalemia. The common risk factors of hyperkalemia include acute or chronic renal insufficiency, medications interfering with urinary potassium excretion, and excessive intake of potassium containing diets [10].

Hyperkalemia can result in life-threatening cardiac arrhythmias and patients with underlying renal impairment would be predisposed to its development. Prompt and aggressive management with medical therapy and hemodialysis could be life-saving in patients with severe hyperkalemia Presented with marked arrhythmia and hemodynamic instability. 


\section{References}

1 Acker CG, Johnson JP, Palevsky PM, Greenberg A (1998) Hyperkalemia in hospitalized patients: causes, adequacy of treatment, and results of an attempt to improve physician compliance with published therapy guidelines. Arch Intern Med 158: 917-924.

2 Gogas BD, Iliodromitis EK, Leftheriotis DI, Flevari PG, Rallidis LS, et al. (2011) Instantaneous electrocardiographic changes and transient sinus rhythm restoration in severe hyperkalemia. Int J Cardiol 148: e40-e42.

3 McIntyre WF, Femenía F, Arce M, Pérez-Riera AR, Baranchuk A (2011) Importance of early electrocardiographic recognition and timely management of hyperkalemia in geriatric patients. Exp Clin Cardiol 16: 47-50.

4 Nyirenda MJ, Tang JI, Padfield PL, SeckI JR (2009) Hyperkalemia. BMJ 339: b4114.
5 Alfonzo AV, Isles C, Geddes C, Deighan C (2006) Potassium disordersclinical spectrum and emergency management. Resuscitation 70: 10-25.

6 Szerlip HM, Weiss J, Singer I (1986) Profound hyperkalemia without electrocardiographic manifestations. Am J Kidney Dis 7: 461-465.

7 Esposito C, Bellotti N, Fasoli G, Foschi A, Plati AR, et al. (2004) Hyperkalemia-induced ECG abnormalities in patients with reduced renal function. Clin Nephrol 62: 465-468.

8 Mattu A, Brady WJ, Robinson DA (2000) Electrocardiographic manifestations of hyperkalemia. Am J Emerg Med 18: 721-729.

9 Weisberg LS (2008) Management of severe hyperkalemia. Crit Care Med 36: 3246-3251.

10 Lehnhardt A, Kemper MJ (2011) Pathogenesis, diagnosis and management of hyperkalemia. Pediatr Nephrol 26: 377-384. 